\title{
Numerical Study of Combined Drag Reduction Bases on Vortex Generators and Riblets for the Ahmed Body using IDDES Methodology
}

\author{
X. Yang, Y. Hư , Z. Gong, J. Jian and Z. Liu \\ State Key Laboratory of Advanced Design and Manufacturing for Vehicle Body, Hunan University, \\ Changsha, Hunan, 410082, China \\ †Corresponding Author Email: 897419969@qq.com
}

(Received March 29, 2021; accepted August 17, 2021)

\begin{abstract}
The flow fields around vehicle drag reduction devices are three-dimensional, complicated, and unsteady. Meanwhile, the small scale of the drag reduction device brings more challenges to the simulation. An Improved Delayed Detached Eddy Simulation (IDDES) was applied to study the effect of two types of drag reduction device. First, the flow field of an Ahmed body at a $25^{\circ}$ slant was simulated in detail using IDDES and verified by experiment data. Afterwards, the flow field structures of adding-on vortex generators (VGs) and riblets on the rear and slant surface of the Ahmed body were studied. The simulation results of the velocity, pressure and vortex structure on the controlled case are presented by comparing with the baseline model to illustrate the drag reduction mechanism. The two drag-reduction devices had different flow mechanisms and significantly affected the development of the separation vortex near the rear and slanted surface. A maximum drag reduction of $6.21 \%$ could be achieved using VGs on the rear surface. Finally, four combinations of those two devices were investigated. The results demonstrated that suitable combinations can further decrease the aerodynamic drag, and an $8.62 \%$ drag reduction is achieved.
\end{abstract}

Keywords: Passive flow control; Vortex generators; Riblets; Combined flow control; Detached eddy simulation.

\section{NOMENCLATURE}

$\begin{array}{ll}A & \text { projected frontal area } \\ C_{d} & \text { drag coefficient } \\ C_{p} & \text { pressure coefficient } \\ C_{f} & \text { frictional drag coefficients } \\ H & \text { height of car model } \\ L & \text { length of car model } \\ m & \text { meter } \\ S & \text { second } \\ W & \text { weight of car model }\end{array}$

\section{INTRODUCTION}

Energy savings and emission reductions are identified as two worldwide issues. In the automotive industry, the reduction of aerodynamic drag is considered an effective method of decreasing fuel consumption and thus saving energy (Wood 2006) which has become an investigative hotspot in recent years. As it well known, pressure drag is formed due to the flow separation at the rear of the vehicle, which is a major contribution to the aerodynamic drag. Therefore, delaying or inhibiting the development of

$\begin{array}{ll}k & \text { turbulent kinetic energy } \\ t & \text { time } \\ \mu & \text { laminar kinematic viscosity } \\ \mu_{T} & \text { turbulent kinematic viscosity } \\ \rho & \text { density } \\ V & \text { wind velocity } \\ V G & \text { vortex generator } \\ R e & \text { Reynolds number }\end{array}$

separation is necessary to reduce the aerodynamic drag. Different flow control strategies have been applied in simplified vehicle models, as shown in the review (Choi et al. 2008, Choi et al. 2014).

Vehicle flow control strategies can be divided into two types, active technology and passive technology. Active techniques employ either blowing (Park et al. 2013, Minelli et al. 2016), or suction (Schatzman et al. 2014). However, active drag reduction devices have a complex control structure and consume extra energy, making their application limited. Passive technology is simple and requires no additional 
energy. It has attracted great research interest and is quite effective for flow control, such as flaps (Altaf et al. 2014; Beaudoin and Aider 2008), span-wise waviness (Lam et al. 2004; Lam and Lin 2007; Zou et al. 2013) and wake splitter plates (Bao and Tao 2013; Gilliéron and Kourta 2010; Rouméas et al. 2009; Capone and Romano 2019). These passive technologies can achieve significant drag reduction effects (Choi et al. 2014). However, they have a comparatively larger mechanical structure and have limited industrial applications. Some researchers have also investigated small size devices, such as vortex generators (VGs) and riblets. VGs and riblets are smaller and are adapted to the law regulations of the practical use. Different VGs studies have been conducted through experiments and numerical simulations (Aider et al. 2010; Krajnović 2014; Pujals et al. 2010; Selvaraju and Parammasivam 2019; Shankar and Devaradjane 2018). The coherent and longitudinal vortex structures along the streamwise direction are generated by the influence of VGs; such vortices push high-momentum fluid to the near-wall region. The pressure gradient is sustained with the enhancement of momentum in this region and the boundary layer separation is delayed or suppressed. Different types of riblets such as rectangular and $\mathrm{V}$-shaped are also applied to achieve passive flow control (Dean and Bhushan 2012; Garcia-Mayoral and Jimenez 2011; WALSH and LINDEMANN 1983; WALSH and LINDEMANN 1984; García-Mayoral and Jiménez 2011; Grüneberger and Hage 2011). The peaks of the riblets can obstruct the spanwise movement of the turbulent flow and cause a secondary vortex, thus reducing turbulent bursts near the surface of the riblets. Additionally, the non-surface contact area is reduced compared to the smooth surface, which improves the tribological performance. In addition, the combination of different flow control strategies can further maximize the flow control effect (Altaf et al. 2014; Wassen and Thiele 2009). Altaf studied the combinations of perforations and different shapes of flaps on a truck model, which obtained better drag reduction compared to unperforated flaps. The present study concerns a single flow control strategy and combines the flow control strategies of VGs and riblets.

While the flow control effectiveness can typically be verified by experiment and numerical simulation, the structural scale of VGs and riblets is small and the flow field around small drag-reduction devices brings great challenge to the numerical simulation. Furthermore, the flow around the vehicle is inherently three dimensional, unsteady and complex. In terms of the engineering problem, a highly accurate turbulence model with a relatively small computation cost is required (Spalart and McLean 2011). In the unsteady flow field, a Reynoldsaveraged Navier-Stokes (RANS) turbulent model cannot accurately predict small-scale unsteady flow (Chok et al. 1994; Guilmineau 2008). Large eddy simulation (LES) can be applied to successfully predict the wake flow field around the Ahmed model. Additionally, it can be used to predict flow control problems (Krajnović and Basara 2010; Serre et al. 2013). However, due to the requirement for very fine grids, LES needs a lot of computational effort. Hybrid RANS-LES models have been proposed and used in flow prediction (Spalart 1997; Spalart et al. 2006). Hybrid methods can reduce the computational cost of the near-wall simulation and save computational time. At the same time, it can offer certain advantages of the LES model in the separated region. Guilmineau investigated the external flow field of the Ahmed body using the Detach Eddy Simulation (DES) turbulent model (Guilmineau et al. 2018). The hybrid RANS-LES models were also applied in the flow control problem. The Very Large Eddy Simulation (VLES) model is used to simulate the flow field around a Simplified D-Shaped Cylinder (Han and Krajnović 2013). In the present study, IDDES model was applied to the prediction of the flow control problem. IDDES improved the issue that a mismatch exists between the modelled region and the resolved region in the traditional hybrid model, which improves the turbulent model's robustness.

To save computation time and decrease the geometrical complexity, a simplified 3D vehicle model is generally used as the study object. Several prototype models are available, such as the Ahmed body (Ahmed et al. 1984), GM (Han et al. 1996), and GTS (Croll et al. 1995; Gutierrez et al. 1995). The Ahmed body has typical flow characteristics, which was studied experimentally (Ahmed et al. 1984; Lienhart et al. 2003; Conan et al. 2011) and numerically (Chok et al. 1994; Guilmineau et al. 2011; Guilmineau et al. 2018). The main pressure drag of the Ahmed body is derived from the wake region and it has different flow structures at different slant angles. Most previous studies have focused on the Ahmed body at a $25^{\circ}$ slant angle and $35^{\circ}$ slant angle (Chok et al. 1994; Guilmineau 2008; Guilmineau et al. 2011). For the $35^{\circ}$ slant angle case, the Ahmed body is completely separated from a large vortex in the wake region. The wake flow structure of the Ahmed model at $25^{\circ}$ is characterized by threedimensional flow separation and reattachment occurs on the slant surface, which makes it more complicated and difficult to accurately predict the flow field. Therefore, studying the flow control of the Ahmed body at $25^{\circ}$ remains a challenge.

In the current work, the natural flow of the Ahmed body at a $25^{\circ}$ slant was accurately predicted first using the IDDES model. Then, the flow fields of the Ahmed body with two flow control devices were numerically studied. This study is organized as follows. Section 2 describes the details of the geometry and mesh and Section 3 gives a brief review of the numerical method. Section 4 provides the simulation results for the Ahmed body with the hemispherical VGs, longitudinal riblets, and their combinations. Section 5 presents the conclusion.

\section{GEOMETRY}

The Ahmed model is chosen as the study object. Figure 1 presents the geometry of the Ahmed body (Ahmed et al. 1984), which is a symmetrical bluff with rounded front surfaces and a rear slant surface. The length of the Ahmed body is $1,044 \mathrm{~mm}$; other 
geometric quantities are normalized with the body length. The body width is $\mathrm{W} / \mathrm{L}=0.373$, the body height is $\mathrm{H} / \mathrm{L}=0.276$, and the radius of the rounded front part is $\mathrm{R} / \mathrm{L}=0.096$. The slant angle is $25^{\circ}$ and the slant length is $\mathrm{S} / \mathrm{L}=0.213$. As is well-known, setting a proper blocking rate is very important for accurately predicting the flow field (Keogh et al. 2016). In this study, the computational domain is $\Omega=10 \mathrm{~L} \times 7 \mathrm{~W} \times 5 \mathrm{H}$ and the blocking factor is $2.49 \%$. The front face of the Ahmed body is located at $3 \mathrm{~L}$ away from the domain inlet and the distance from the rear surface to the outlet is $6 \mathrm{~L}$.
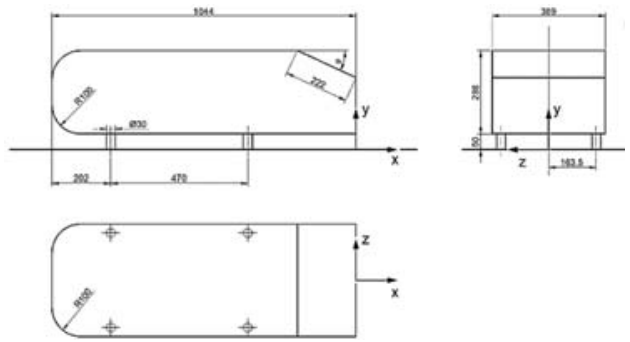

Fig. 1. Side, rear and top view of the Ahmed body, dimensions are given in millimeter.

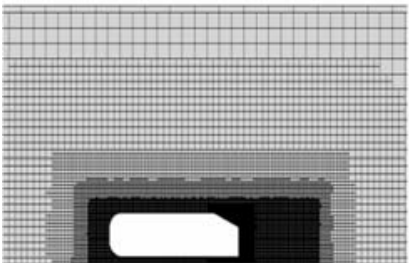

(a)

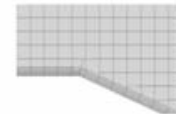

(b)

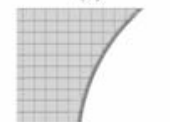

(c)
Fig. 2. Mesh around Ahmed body: (a)Global view; (b)Local view of the slant; (c) Local view of the front.

The computation domain is discretized by the trimmer meshes. Figure 2 shows a global view of the mesh distribution on the symmetry plane. Note that the prism layers were only extruded from the Ahmed body surface to model the boundary layer around it. Volumetric control boxes with finer meshes were used at the front face and at the back of the Ahmed body. To test the grid independence, three sets of computational mesh were used, which are listed in Table 1.

\section{NUMERICAL MODEL AND SETUP}

To capture the unsteady and small-scale flow around the drag reduction device, the IDDES model was adopted that was based on the k-omega model (Shur et al. 2008; Gritskevich et al. 2012). IDDES combines near-wall RANS modelling with far-wall LES modelling for applications such as high-Re external aerodynamics simulations, thus endowing it with high accuracy and low computation cost.
Table 1 Characteristics of computational grids

\begin{tabular}{|c|c|c|}
\hline Configurations & grid number & $\begin{array}{c}\text { Y plus of } \\
\text { wall }\end{array}$ \\
\hline Coarse mesh & 6.8 million & 15 \\
\hline Medium mesh & 12.6 million & 10 \\
\hline Fine mesh & 23.1 million & 1 \\
\hline
\end{tabular}

The IDDES model modifies the sink term in the $\mathrm{k}$ equation of the SST k- $\omega$ model.

$$
\begin{gathered}
\begin{aligned}
\frac{\partial \rho k}{\partial t}+\nabla \cdot(\bar{\rho} \widetilde{U} k) & =\nabla \cdot\left[\left(\mu+\frac{\mu_{T}}{\sigma_{k 3}}\right) \nabla k\right]+P_{k} \\
& -\rho \beta^{*} k \omega F_{I D D E S}
\end{aligned} \\
\begin{aligned}
& \frac{\partial \rho \omega}{\partial t}+\nabla \cdot(\bar{\rho} \widetilde{U} \omega)=\nabla \cdot\left[\left(\mu+\frac{\mu_{T}}{\sigma_{\omega 3}}\right) \nabla \omega\right]+(1- \\
&\left.F_{1}\right) 2 \rho \frac{\nabla k \nabla \omega}{\sigma_{\omega 2} \omega}+\alpha \frac{\omega}{k} P_{k}-\beta_{3} \rho \omega^{2}
\end{aligned}
\end{gathered}
$$

Where $F_{I D D E S}$ is defined as:

$F_{I D D E S}=\frac{l_{R A N S}}{l_{I D D E S}}$

$F_{I D D E S}$ is a trigger filter and $l_{I D D E S}$ is the function enables the solver to automatically identify the RANS region and the LES region and realize the switch from RANS to LES. It can be defined as:

$l_{I D D E S}=\tilde{f}_{d}\left(1+f_{e}\right) l_{R A N S}+\left(1-\tilde{f}_{d}\right) l_{L E S}$

Where $\tilde{f}_{d}=\max \left[1-f_{d t}, f_{B}\right]$.Variables such as $f_{d t}, f_{B}, f_{d}$ and $f_{e}$ can be referenced from Gritskevich et al. (2012), $\mathrm{F}_{\text {IDDES }}$ is related to $l_{R A N S}$ and $l_{L E S}, l_{R A N S}$ and $l_{L E S}$ can be defined as:

$l_{R A N S}=\frac{\sqrt{k}}{\beta^{*} \omega}$

$l_{L E S}=C_{D E S} \Delta$

$l_{R A N S}$ is related to the $k$ and $\omega$ items. $l_{L E S}$ is affected by the grid scale and $C_{D E S}$ is a DES model constant. In the IDDES model, the sub-grid scale needs to consider the influence of the local grid scale and wall distance, which can be expressed as:

$\Delta=\min \left[\max C_{w}\left(\Delta_{\max } ; d\right) ; \Delta_{\max }\right]$

$\Delta_{\max }=\max (\Delta x, \Delta y, \Delta z)$

The present study employed the finite volume method. The IDDES model was implemented in the general CFD code ANSYS FLUENT. The bounded second-order center difference scheme is used to discretize the convection terms. The time advance uses the second-order implicit scheme while the turbulent equation uses the second-order upwind scheme. Pressure-velocity coupling uses the SIMPLEC scheme.

A uniform stream velocity $U_{o}=40 \mathrm{~m} \cdot \mathrm{s}^{-1}$ is used at the inlet that results in Reynolds number $R e_{L}=2.88 \times 10^{6}$ based on the body length and inlet velocity. The outlet boundary pressure is set to be equivalent to the atmospheric pressure. No-slip boundary conditions are used for the surfaces of the Ahmed body and the floor of the computational domain, while the channel lateral surfaces and 
ceiling are treated as slip walls. Both the natural flow and controlled flow of the Ahmed model are studied. The time step is set to: $\Delta \mathrm{t}=2 \times 10^{-4} \mathrm{~s}$. Meanwhile, the average time $\mathrm{t} U_{o} / L$ is approximately 40 .

\section{RESULTS AND DISCUSSIONS}

\subsection{The baseline Ahmed body with $25^{\circ}$ slant}

To verify the accuracy of the numerical model and the independence of the computational grid, the baseline Ahmed body at a $25^{\circ}$ slant without flow control is analyzed first by employing the IDDES turbulent model. The velocity profile, vortex structure, aerodynamic drag coefficient and turbulent energy at the wake flow field are studied in detail. The results of three computational meshes are verified by comparison with the experimental results (Lienhart et al. 2003; Conan et al. 2011). In addition, the numerical results of the RANS methodology (k$\omega \mathrm{SST}$ ) are used for comparison.

The vortex structure of the wake flow can be shown by the velocity streamline (Anderson $\mathrm{Jr}, 2010$ ). Figure 3 shows the streamlines based on the timeaveraged velocity in the symmetry plane using IDDES with three meshes. The result of unsteady RANS is also included for comparison. Obviously, all turbulence models can be applied to predict the large separation bubble near the rear surface wall. However, the RANS methodology cannot predict the small separation bubble on the slant surface as in Fig. 3(a). For the medium and fine meshes, the IDDES turbulence model has a successful prediction. The small separation and reattachment are reproduced on the slant surface as shown in Fig. 3(c) and 3(d), which are consistent with the experimental results (Ahmed et al. 1984).

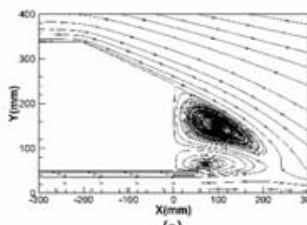

(a)
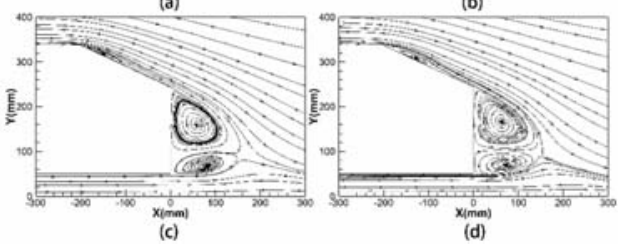

Fig. 3. Comparison of streamlines based on timeaveraged velocity in symmetry plane $Z=0$ : (a) $k$ w SST; (b) Coarse mesh - IDDES; (c) Medium mesh - IDDES; (d) Fine mesh - IDDES.

To further verify the model and the grid independence in quantitative analysis, Fig. 4 presents a comparison of the time-averaged streamwise velocity profiles along the $\mathrm{x}$-direction near the slant surface in the symmetry plane. Again, the results of the IDDES model are consistent with the experimental values (Lienhart et al. 2003) well. The medium-mesh results are very close to the fine-mesh results. The RANS methodology shows an obvious difference when compared with the experimental values (Fig. 4).

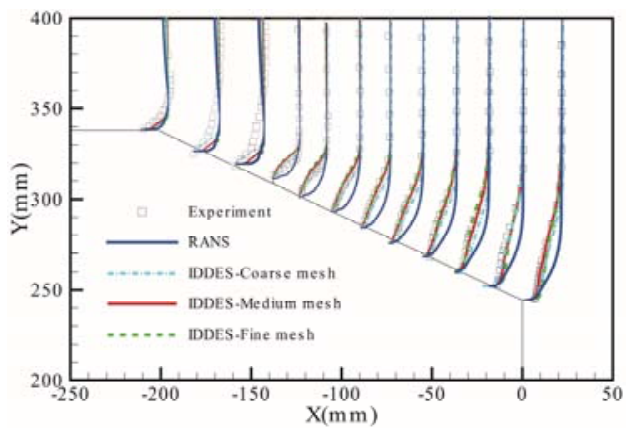

Fig. 4. Comparison of time-averaged streamwise velocity profiles on the wake region in symmetry plane $\mathbf{Z}=\mathbf{0}$.

Figure 5 presents a comparison of the RMS streamwise velocity (Urms/Uo), which can reflect flow fluctuations in the wake. Obviously, there are great differences between the two turbulence models. For the RANS methodology, there are only velocity fluctuations near the rear region of the Ahmed body, whereas for the IDDES model, velocity fluctuations can be found in both the region near the slant surface and the rear region of the Ahmed body. Compared with the coarse mesh, the results of the medium mesh and the fine mesh are similar on the slant region, but there is a little difference at the bottom region.

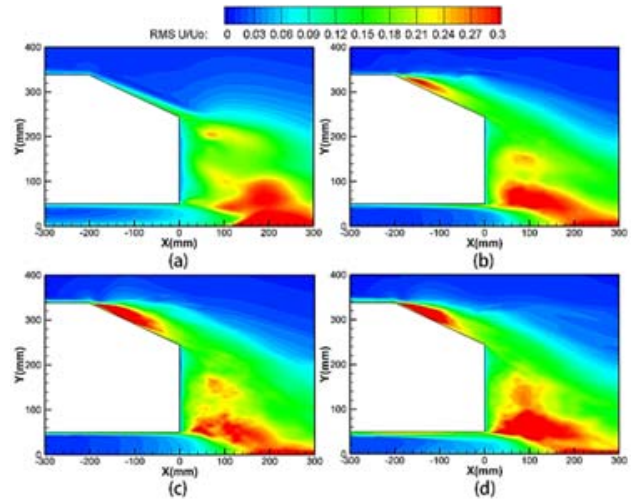

Fig. 5. Comparison of the rms streamwise velocity Urms/Uo on the wake region in $Z=0$

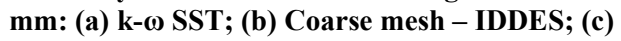
Medium mesh - IDDES; (d) Fine mesh - IDDES.

The drag coefficient is an important parameter that is used to reflect the aerodynamic characteristics of the Ahmed body. Table 2 demonstrates the drag coefficients of RANS, IDDES and experimental measurement data (Conan et al. 2011). The 
separation bubble on the slant surface cannot be predicted by the RANS methodology, which results in an underestimated pressure drag and a relatively low drag coefficient. The drag coefficient obtained by the IDDES model is higher than the experiment value (Ahmed et al. 1984). One thing worth noting is that the drag coefficient measured by Ahmed based on a Reynolds number of 4.2 million, which is higher than that in the present study. The Reynolds number has a considerable impact on the flow structure and may explain the relatively high drag coefficient for IDDES. However, the drag coefficient obtained with the IDDES model agrees well with another experiment (Conan et al. 2011) that has a closer Reynolds number of 2.86 million. In the prediction of the drag coefficient, the coarse mesh shows larger difference than other two meshes while the difference between the medium grid and the fine grid is small, approximately $0.9 \%$. Thus, a medium mesh was used in the following study to reduce the computation cost and improve the simulation efficiency.

Table 2 Drag coefficients of Ahmed body at $25^{\circ}$ slant

\begin{tabular}{|c|c|}
\hline Configurations & Drag coefficient \\
\hline Coarse grid- IDDES & 0.3257 \\
\hline Medium grid- IDDES & 0.3401 \\
\hline Fine grid-IDDES & 0.3432 \\
\hline RANS & 0.2974 \\
\hline Experiment & 0.3443 \\
\hline
\end{tabular}

\subsection{Controlled flow with VGs}

As vortex generators, the cylindrical roughness elements and the small blade are applied to control the wake flow (Aider et al. 2010; Pujals et al. 2010). In the current work, which investigates a new type of $\mathrm{VG}$, hemispherical round roughness elements were applied to both the slant surface and the rear surface of the Ahmed body (see Fig. 6(a) and 6(b)). Compared to cylindrical roughness elements, the hemispherical elements can also produce similar small-scale coherent vortices; however, it has a comparatively simpler structure and smaller volume. Figure 6(c) shows the geometry parameters of VGs and Fig. 7 shows the local mesh cuts of VGs. The VGs in Fig. 6 have 10 round roughness elements, with $\mathrm{L} 1=35 \mathrm{~mm}, \mathrm{D} 1=$ $20 \mathrm{~mm}, \mathrm{~L} 2=35 \mathrm{~mm}, \mathrm{D} 2=20 \mathrm{~mm}$. To predict the detailed flow around the VGs, a smaller grid size is applied to the local region of VGs. The total number of mesh cells is approximately 18 million for VGs on the slant surface and 16 million mesh cells for VGs on the rear surface, both of which are based on the original medium mesh with 12.6 million mesh cells. It should be pointed out that the practical instantaneous flow structure is complicated and changes over time. Therefore,

most analyses concentrate on the time-averaged results including velocity, pressure coefficient, vortex structure, wake dynamic and drag coefficient. These results are examined and compared with the original flow without any control.

Figure 8 shows the time-averaged velocity streamline around the Ahmed body for three cases: (a) the baseline Ahmed body, (b) VGs added on the slant surface, and (c) VGs added on the rear surface. To quantify the effect of the vortex on the wake region, the center of the three separation bubbles is marked on Fig. 8. Besides, Table 3 presents its position in the $\mathrm{X}$ direction. Evidently, there are two separation zones in Fig. 8(a) for the baseline Ahmed body, which is the same as Fig. 3. In contrast, for the case of VGs on the slant surface, the small separation on the slant is disturbed in Fig. 8(b) and cannot form a significant separation bubble. The small separation bubble is unaffected by adding VGs at the rear. However, the separation of the two large vortices at the rear region is delayed, as is shown in Fig. 8(c). The separation bubble moves away from the rear vertical plane and the reattachment length of the near-wake separation bubble is increased. The separation delay may increase the pressure near the rear wall and eventually reduce the aerodynamic drag.

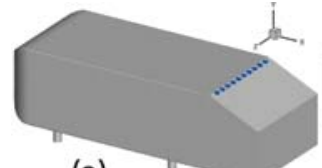

(a)
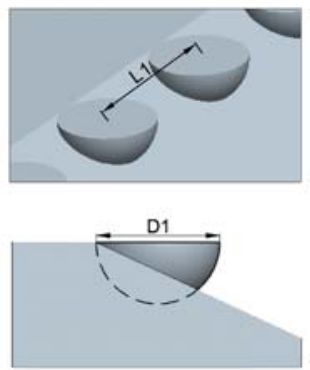

(c)

Fig. 6. Ahmed body with VGs: (a) the global view of VGs added on slant surface; (b) the global view of VGs added on rear surface; (c) local view and the relevant parameter of VGs.

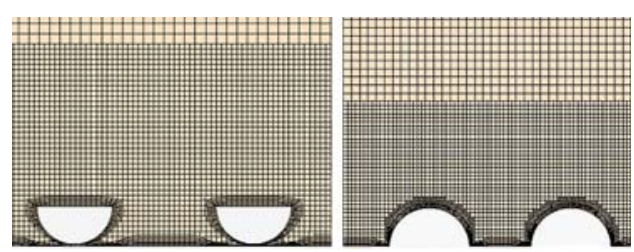

Fig. 7. local view of the VGs mesh cut. 
Table 3 The position of wake vortex controlled by VGs, dimensions are given in millimeters

\begin{tabular}{|c|c|c|c|}
\hline \multirow{2}{*}{ Configuration } & \multicolumn{3}{|c|}{ X-direction position of the wake vortex } \\
\cline { 2 - 4 } & Slant vortex & Rear vortex 1 & Rear vortex 2 \\
\hline Baseline Ahmed body & -144.53 & 58.01 & 82.22 \\
\hline VGs added on slant surface & None & 64.62 & 81.49 \\
\hline VGs added on rear surface & -130.59 & 80.76 & 91.13 \\
\hline
\end{tabular}

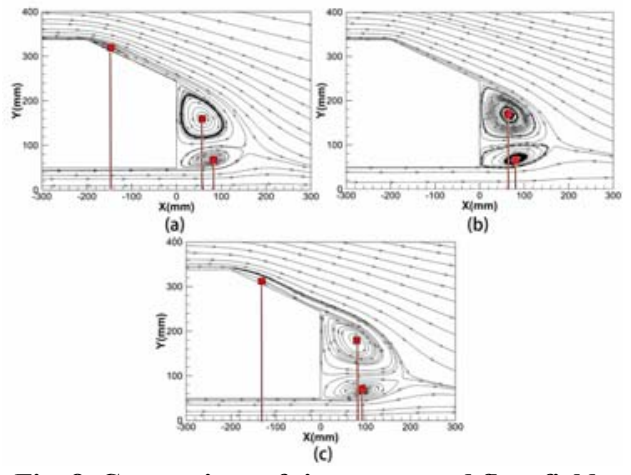

Fig. 8. Comparison of time-averaged flow fields in symmetry plane $Z=0$ : (a) Baseline Ahmed body; (b) VGs added on slant surface; (c) VGs added on rear surface.
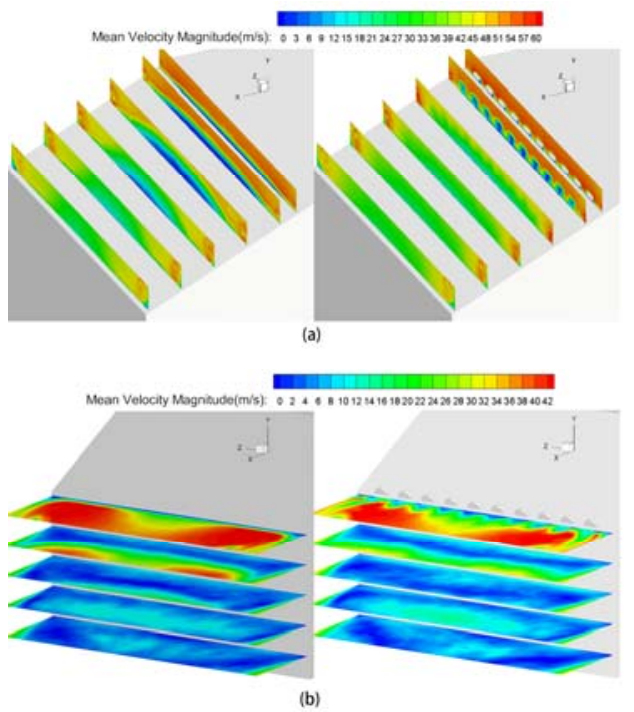

Fig. 9. Comparison of time-averaged streamwise velocity at different plane positions: (a) Baseline Ahmed body(left) and VGs added on slant surface(right); (b) Baseline Ahmed body(left) and VGs added on rear surface(right).

To further study the detailed flow around the VGs, Fig. 9 shows comparisons of the time-averaged velocity magnitude for the natural case and the controlled cases in the spanwise plane. Figure 10 shows the velocity streamline at the slant and rear surface. Additionally, the local streamline distribution near the VGs is shown in a corresponding enlarged view. Figure 9 (a) is the case with VGs on a slant, which is compared with the natural flow. Six sections are selected along the $\mathrm{x}$ axis: $X=-185 \mathrm{~mm},-165 \mathrm{~mm},-125 \mathrm{~mm},-85 \mathrm{~mm},-45$ $\mathrm{mm}$, and $-5 \mathrm{~mm}$. Obviously, large low-speed zones were clearly observed for the natural flow case on the slant; and it shows the approximate length of the separation bubble in the streamwise and spanwise direction. However, when VGs were added on the slant surface, the low-speed zone at $X=-185 \mathrm{~mm}$ and $X=-165 \mathrm{~mm}$ sections was broken into several small zones around the VGs. And at the following slant planes, the low-speed zones disappeared, indicating that the separation bubble was effectively reduced in the slant surface. The same phenomenon can be found clearly in Fig. 10(b). Due to the influence of VGs, the coherent small vortex structure is generated after the hemisphere. With the further development of these vortices, the formation of the separation bubble on the slant is disturbed compared to the natural flow in Fig. 10(a). For the case in which VGs are added on the rear surface, five sections are selected along the $y$-axis at $Y=240 \mathrm{~mm}, Y=200$ $\mathrm{mm}, \mathrm{Y}=160 \mathrm{~mm}, \mathrm{Y}=120 \mathrm{~mm}$ and $\mathrm{Y}=80 \mathrm{~mm}$ to show the difference between controlled flow and natural flow (Fig. 9(b)). Compared with the natural flow, the low-speed zone is also generated in the near region of the VGs. At $\mathrm{Y}=200 \mathrm{~mm}$ and $\mathrm{Y}=160 \mathrm{~mm}$, the low-speed streak moves away from the vertical plane of the Ahmed body. Figure 10(c) shows the streamline in detail. The wake flow near the rear region is affected by the coherent vortices generated by VGs. Based on this, the main wake vortex core moves away from the rear wall as shown in Fig. 8(c).

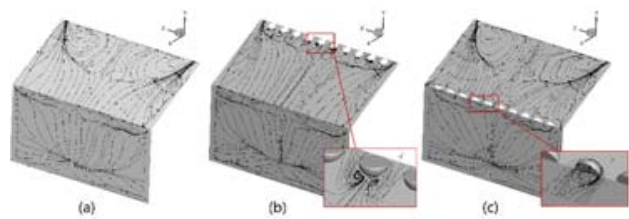

Fig. 10. Comparison of surface streamlines on the wake region: (a) Baseline Ahmed body; (b) VGs added on slant surface; (c) VGs added on rear surface.

Figure 11 compares the predictions of isosurfaces of the $\mathrm{Q}$ criterion in the wake region between the natural flow and controlled flow with VGs, demonstrating the development of $3 \mathrm{D}$ vortices in the wake region. The ISO-surface value of $\mathrm{Q}$ is set to 
X. Yang et al. / JAFM, Vol. 15, No. 1, pp. 193-207, 2022

Table 4 Comparison of drag coefficients with VGs flow control

\begin{tabular}{|c|c|c|}
\hline Configurations & $\begin{array}{c}\text { Total drag } \\
\text { coefficients }\end{array}$ & $\begin{array}{c}\text { Total drag } \\
\text { reduction }\end{array}$ \\
\hline $\begin{array}{c}\text { Baseline Ahmed } \\
\text { body }\end{array}$ & 0.3401 & - \\
\hline $\begin{array}{c}\text { VGs added on } \\
\text { slant surface }\end{array}$ & 0.3250 & $4.37 \%$ \\
\hline $\begin{array}{c}\text { VGs added on rear } \\
\text { surface }\end{array}$ & 0.3193 & $6.21 \%$ \\
\hline
\end{tabular}

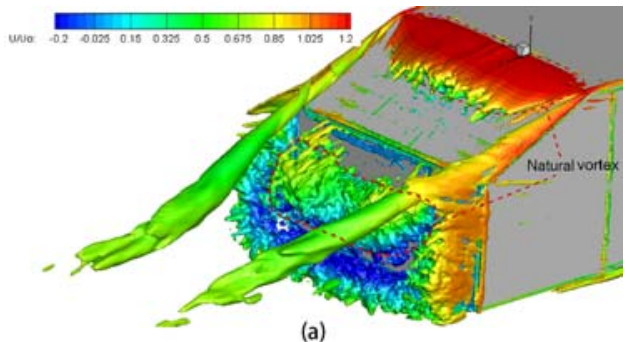

(a)
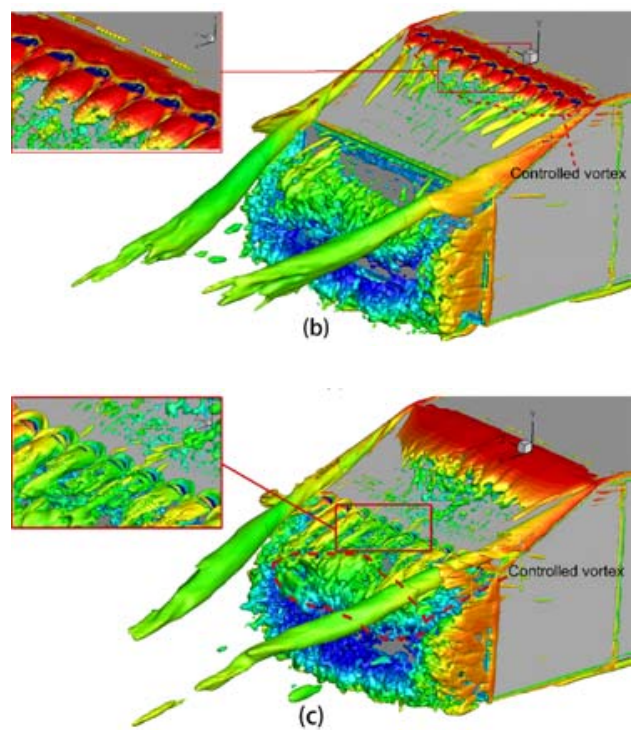

Fig. 11. Comparison of ISO-surface of timeaveraged $Q$ criterion in the wake region: (a) Baseline Ahmed body; (b) VGs added on slant surface; (c) VGs added on rear surface.

50,000 to capture the flow structure. As shown in Fig. 11(a), small turbulent structures are generated. In Fig. 11(b), VGs are applied on the slant surface. Small vortices generated by VGs are developed that disturb the formation of the separation bubble on the slant surface. This increases the pressure on the slant surface and reduces the drag. Similarly, Fig. 11(c) clearly shows that small vortices generated by the rear VGs interfere with the wake vortices, resulting in a large vortex structure that is transformed into a smaller vortex structure. Consequently, the strength of the wake vortices is reduced and the pressure is increased in the rear surface.

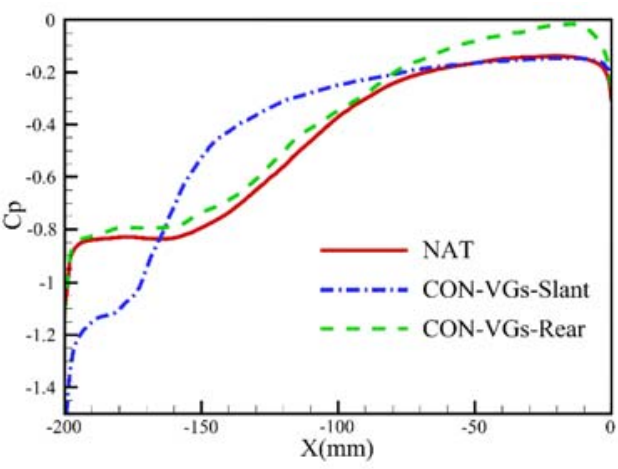

(a)

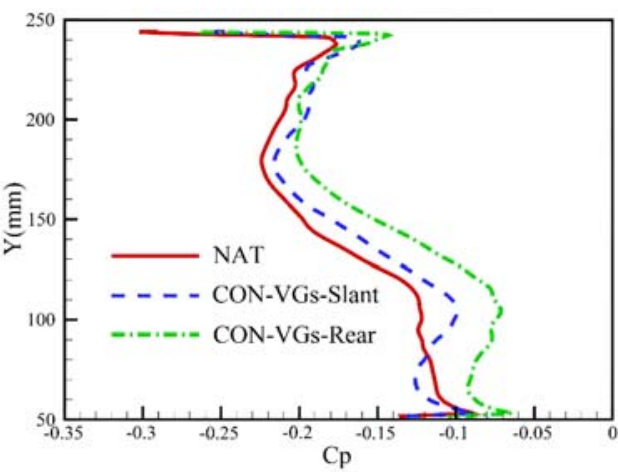

(b)

Fig. 12. Comparison of the surface pressure coefficient $\mathrm{Cp}$ between natural flow and the controlled flow using VGs: (a) Slant surface; (b) Rear surface.

The aerodynamic drag is significantly affected by the pressure distribution around the Ahmed body. Figure 12 shows a comparison of the surface pressure coefficient $\mathrm{Cp}$, which mainly illustrates the timeaveraged pressure distribution on the slant surface (Fig. 12(a)) and rear surface (Fig. 12(b)). As can be seen in Fig. 12(a), the pressure coefficient for the natural flow increases initially until it reaches its maximum value and then has a decreasing trend. The dashed line corresponds to the surface pressure coefficient with VGs added on the slant surface. It can be found that the $\mathrm{Cp}$ drops initially. The reason for this is that the velocity increases while the pressure decreases when the incoming flow is passes the gap between VGs. However, the separation 
Table 5 Position of wake vortex controlled by riblets, dimensions are given in millimeters

\begin{tabular}{|c|c|c|c|}
\hline \multirow{2}{*}{ Configuration } & \multicolumn{3}{|c|}{ X-direction position of the wake vortex } \\
\cline { 2 - 4 } & Slant vortex & Rear vortex 1 & Rear vortex 2 \\
\hline Baseline Ahmed body & -144.53 & 58.01 & 82.22 \\
\hline $\begin{array}{c}\text { Riblets added on slant } \\
\text { surface }\end{array}$ & NONE & 42.55 & 63.74 \\
\hline $\begin{array}{c}\text { Riblets added on rear } \\
\text { surface }\end{array}$ & -106.13 & 59.61 & 65.24 \\
\hline
\end{tabular}

bubble of the slant surface is reduced due to the influence of the VGs; thus, the pressure rapidly increases. The pressure coefficient for the case of VGs on the rear surface is shown in the dash-dot line and the $\mathrm{Cp}$ is very close to the natural flow except at the end of the slant surface. Figure 12(b) shows that the effect on the rear pressure coefficient is small for VGs on the slant, which is consistent with the above analysis. When VGs are added on the rear surface, since the large near-wake separation bubble is affected by the VGs, the low-speed zone is decreased, which leads to an obvious increase in the pressure coefficient.

Table 4 shows a comparison of the total drag coefficient and includes the friction coefficient. This clearly shows that both VG cases have good results on drag reduction. The VGs applied on the rear surface have a better drag reduction effect with a decrease of $6.21 \%$

\subsection{Controlled flow with riblets}

As a type of non-smooth surface, $\mathrm{V}$-shape riblets are used as drag reduction devices here. Figure 13(a) and 13(b) show the global view of riblets on the slant surface and rear surface. Besides the experience of experiments (WALSH and LINDEMANN 1984), test simulations are carried out to optimise the geometrical structure of the V-shaped riblet (Zhang et al. 2018). The drag reduction performance of the $\mathrm{V}$-shaped riblet is mainly affected by the riblet's width and depth. A similar configuration (Zhang et al. 2018) is adopted with optimized geometry parameters. The same V-shape riblets were applied to the slant and rear surfaces of the Ahmed body. The local view of the riblets can be seen in Fig. 13(c), where the apex angle is $64^{\circ}, \mathrm{W}=3.8 \mathrm{~mm}$ and $\mathrm{H}=$ $3.2 \mathrm{~mm}$. Due to the small structures of the riblets, a much finer grid is needed near the riblets to obtain the detailed flow structure. Figure 14 shows the local view of the riblets grid. The total number of mesh cells is approximately 24 million for riblets on the slant surface and 22 million mesh cells for riblets on the rear surface based on the original medium mesh. The same analysis as the VGs is carried out in the following sections.

Figure 15 compares the time-averaged streamline in the symmetrical section between the natural flow and controlled flow. Similar to the VGs case, Table 5 also lists the centers of the separation bubbles. Figure 15 (a) is the original case without any control. Figure 15(b) shows the results of riblets added on the slant surface and Fig. 15(c) shows riblets added on the rear surface. As can be seen in Fig. 15(b), similar to VGs,

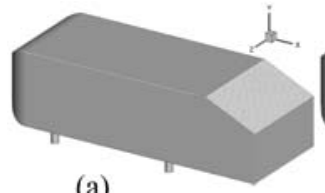

(a)

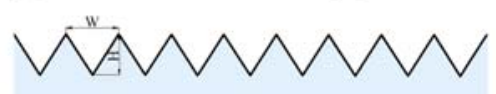

(c)

Fig. 13. Ahmed body with riblets: (a) Global view of riblets added on slant surface; (b) Global view of riblets added on rear surface; (c) local view and the relevant parameter of riblets.

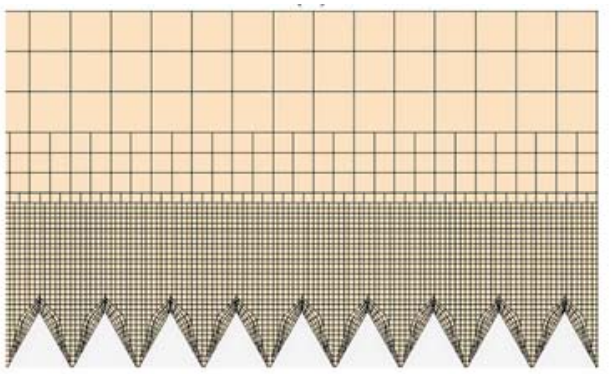

Fig. 14. local view of the riblets mesh cut.

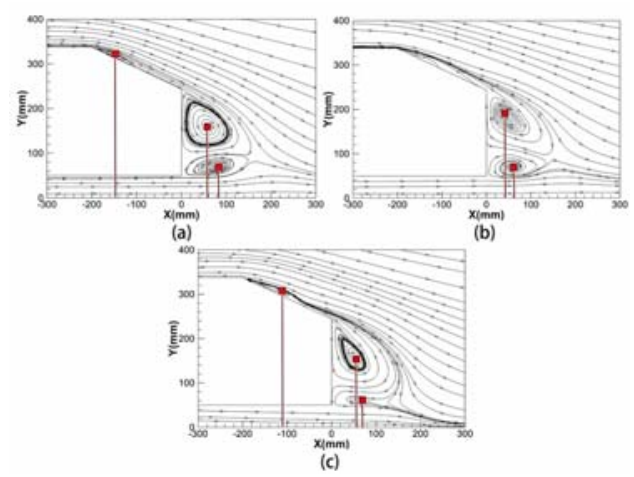

Fig. 15. Comparison of time-averaged flow fields in symmetry plane $Z=0$ : (a) Baseline Ahmed body; (b) Riblets added on slant surface; (c) Riblets added on rear surface.

riblets can affect the small separation bubble on the slant surface. The separation bubble becomes small 
and only exists near the top surface of the Ahmed body. In return, it also affects the formation of the large separation bubble near the rear region. In Fig. 15(c), the riblet influences the formation of the large separation bubble in the near-rear region. Correspondingly, the position of the reattachment point also changes. In particular, the separation bubble at the lower position near the rear region is significantly reduced. In addition, the small separation bubble on the slant surface is also changed and the bubble center is moved a little downstream.

Figure 16 shows the distributions of the timeaveraged velocity magnitude for the natural flow and controlled flow on different sections in the spanwise plane. The position of the section is the same as that in Fig. 9. As shown in Fig. 16(a), the controlled flow by the riblets has a comparatively wider low-speed streak in the spanwise direction than the natural flow at the $X=-185 \mathrm{~mm}$ and $X=-165 \mathrm{~mm}$ planes, resulting in a more uniform velocity distribution.
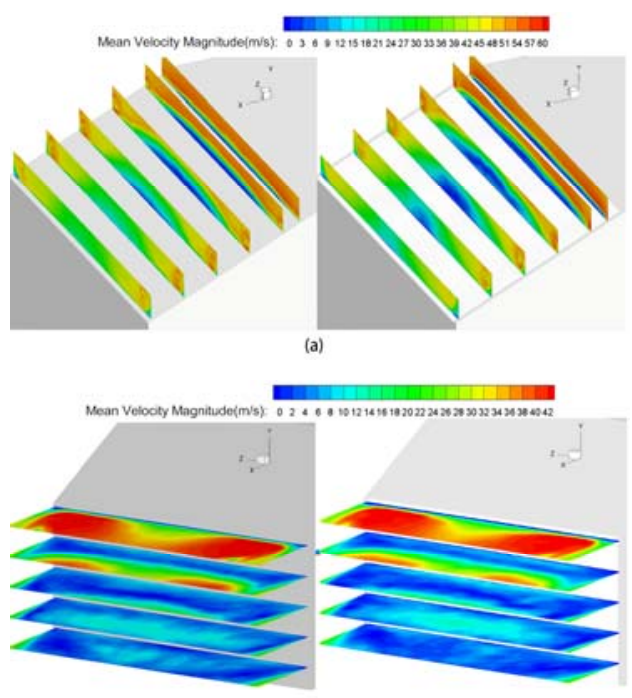

(b)

Fig. 16. Comparison of time-averaged streamwise velocity at different plane positions:

(a) Baseline Ahmed body(left) and riblets added on slant surface(right); (b) Baseline Ahmed body(left) and riblets added on rear surface(right).

Figure 17(a) presents the detail flow for riblets on the slant surface, which shows the velocity streamline and velocity distribution at the $X=125 \mathrm{~mm}$ plane. The flow inside the riblet is shown more clearly by the partial enlargement. Compared with the smooth wall of the natural flow, low-speed streaks and a large number of small vortex structures can be observed inside the riblet. Low-speed streaks and small vortices in the near-wall region can reduce the direct impact of high-speed flow on the solid wall, which can further reduce the flow friction. Meanwhile, Fig. 17(a) shows that the low-speed
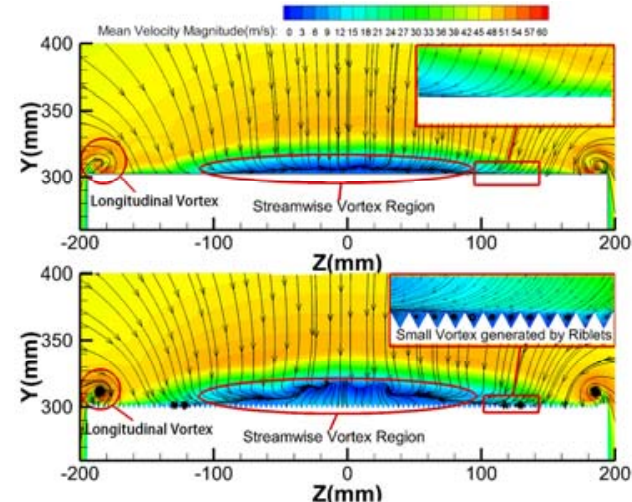

(a)
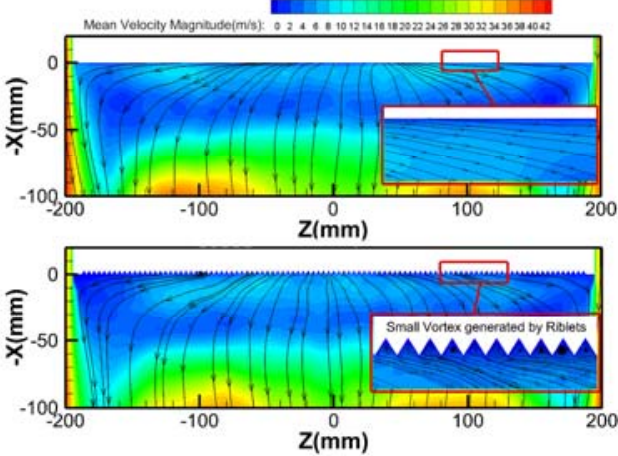

Fig. 17. Comparison of time-averaged streamwise velocity in the local region. The case is organized as: (a) Baseline Ahmed body(upper) and riblets added on slant surface(lower); (b) Baseline Ahmed body(upper) and riblets added on rear surface(lower).

streak moves upwards in the streamwise vortex region, which weakens the effect of the separation bubble on the slant surface and increases the pressure. However, one can predict that the influence on the pressure is weak. For riblets on the rear surface, similar results are shown in Figs. 16(b) and 17(b). Figure 17(b) shows the velocity streak and streamline distribution for the $\mathrm{Y}=200 \mathrm{~mm}$ plane. In the partial enlargement diagram, low-speed streaks and a large number of small vortex structures can be observed inside the riblet, which will result in a similar drag reduction effect as riblets on the slant; friction will be decreased and the pressure will increase slightly in return.

Fig. 18 shows the predictions of the iso-surfaces of the Q criterion, including both the global view and local view at the slant and rear surfaces. The observed position and $\mathrm{Q}$ value are both similar to VGs on Fig. 11. Similarly, Fig. 18(a) is the original Ahmed case. As can be seen from Fig. 18(b), a large number of strip vortexes are generated inside the riblet compared to the natural flow. The local view shows the details of these small vortexes around the riblets. Meanwhile, the generation of small vortices may affect the separation bubbles on the slant surface. Mutual interactions between those vortices 

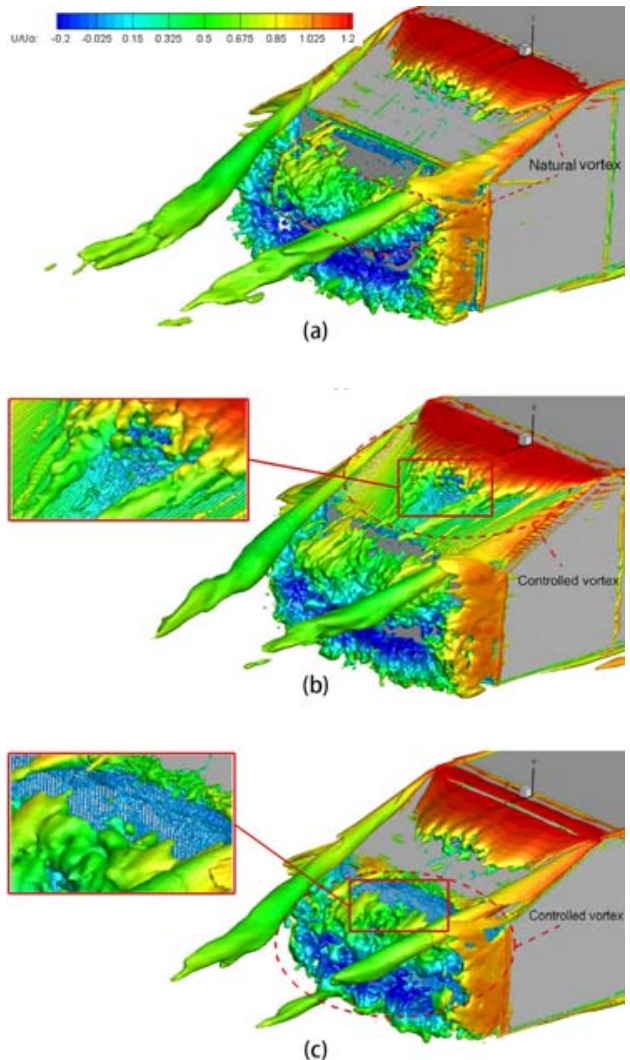

Fig. 18. Comparison of ISO-surface of timeaveraged $Q$ criterion in the wake region: (a)

Baseline Ahmed body; (b) Riblets added on slant surface; (c) Riblets added on rear surface.

may be conductive to reducing the surface friction drag and pressure drag. While in Fig. 18(c), similar to riblets on the slant surface, small vortices are mainly generated at the rear surface and can be observed in a local view.

Figure 19 shows the surface pressure coefficient $\mathrm{Cp}$ for riblets in the symmetry plane of the Ahmed body. The coordinates in Fig. 19 are the same as those in Fig. 12. The dash-dot line is the surface pressure coefficient of the case with riblets on the slant surface. As mentioned before, the separation bubble center moves to the top surface and the strength of the vortices is reduced, which leads to an increase in the pressure coefficient as shown in Fig. 19(a).

However, the effect on the large separation bubble near the rear region is weak. Therefore, the $\mathrm{Cp}$ is

almost the same as the natural flow as Fig. 19(b). The case of riblets added on the rear surface corresponds to the dashed line. As shown in Fig. 15(c), the small bubble center on slant surface is moved downstream slightly. Thus, the $\mathrm{Cp}$ increases a little and then decreases compared to the natural flow. At the rear surface, the $\mathrm{Cp}$ increases again because the large separation bubble near the rear region is weakened by riblets, particularly the lower part. A relatively small increase in the pressure coefficient can be observed in Fig. 19(b).

Figure 19 shows the surface pressure coefficient $C p$ for riblets in the symmetry plane of the Ahmed body. The coordinates in Fig. 19 are the same as those in Fig. 12.

The dash-dot line is the surface pressure coefficient of the case with riblets on the slant surface. As mentioned before, the separation bubble center moves to the top surface and the strength of the vortices is reduced, which leads to an increase in the pressure coefficient as shown in Fig. 19(a). However, the effect on the large separation bubble near the rear region is weak. Therefore, the $\mathrm{Cp}$ is almost the same as the natural flow as Fig. 19(b).

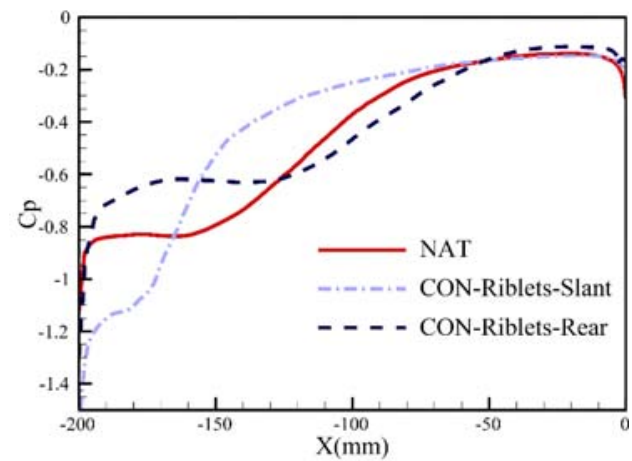

(a)

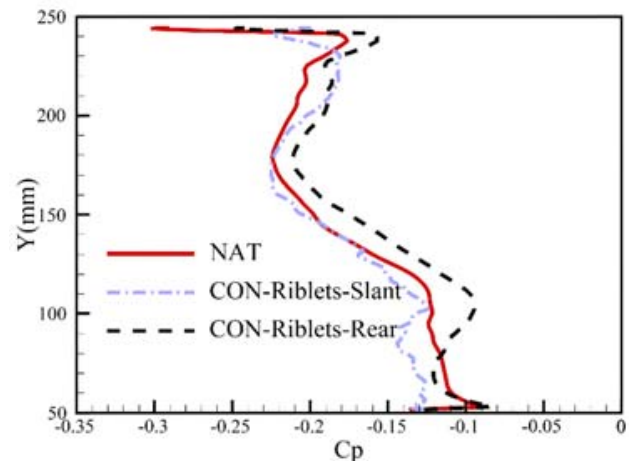

(b)

Fig. 19. Comparison of the surface pressure coefficient $\mathrm{Cp}$ between natural flow and the controlled flow using riblets: (a) Slant surface;

(b) Rear surface.

The case of riblets added on the rear surface corresponds to the dashed line. As shown in Fig. 15(c), the small bubble center on slant surface is moved downstream slightly. Thus, the $\mathrm{Cp}$ increases a little and then decreases compared to the natural flow. At the rear surface, the $\mathrm{Cp}$ increases again because the large separation bubble near the rear region is weakened by riblets, particularly the lower part. A relatively small increase in the pressure coefficient can be observed in Fig. 19(b). 
Table 6 Comparison of drag coefficients with riblets flow control

\begin{tabular}{|c|c|c|c|c|}
\hline Configurations & $\begin{array}{c}\text { Frictional drag } \\
\text { coefficients }\end{array}$ & $\begin{array}{c}\text { Frictional drag } \\
\text { reduction }\end{array}$ & $\begin{array}{c}\text { Total drag } \\
\text { coefficients }\end{array}$ & $\begin{array}{c}\text { Total drag } \\
\text { reduction }\end{array}$ \\
\hline $\begin{array}{c}\text { Baseline Ahmed } \\
\text { body }\end{array}$ & 0.0385 & - & 0.3401 & - \\
\hline $\begin{array}{c}\text { Riblets added on } \\
\text { slant surface }\end{array}$ & 0.0344 & $10.65 \%$ & 0.3326 & $2.21 \%$ \\
\hline $\begin{array}{c}\text { Riblets added on } \\
\text { rear surface }\end{array}$ & 0.0341 & $11.43 \%$ & 0.3298 & $3.03 \%$ \\
\hline
\end{tabular}

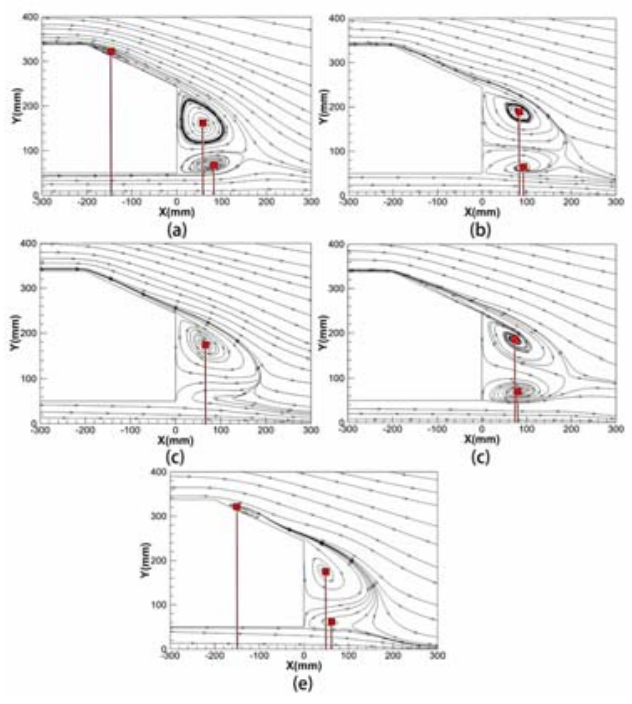

Fig. 20. Comparison of time-averaged flow fields in symmetry plane $Z=0$ : (a) Baseline Ahmed body (b) VGs added on both the slant and rear surfaces; (c) VGs added on the slant surface with riblets added on the rear surface; (d) Riblets added on the slant surface with VGs added on the rear surface; (e) Riblets added on both the slant and rear surfaces.

Table 6 shows the frictional drag coefficients and total drag coefficient using riblets at the slant surface and rear surface, which are compared with the natural flow without control. Evidently, both cases generate good results for the frictional drag reduction, which is $10.65 \%$ and $11.43 \%$ respectively. However, as described by the above analysis, the total drag reduction effect of the riblets is worse than the VGs because the effects of riblets on the large wake vortex are small. The comparatively better drag reduction effect is obtained using riblets on the rear surface with a decreasing of $3.03 \%$.

\subsection{Controlled flow with combined strategy}

The above results show that VGs and riblets have different drag reduction effects at different positions. To further reduce the aerodynamic drag, a combination of those two devices was applied on both the slant and rear surfaces to explore the drag reduction effect. There are four types of VG and riblet combination: VGs added on both the slant and rear surfaces (case b); VGs added on the slant surface with riblets added on the rear surface (case c); riblets added on the slant surface with VGs added on the rear surface (case d); riblets added on both the slant and rear surfaces (case e). The meshes of the combined drag reduction device are similar to a single drag reduction device (Fig. 7 and Fig. 14). The numbers of mesh cells are 21 million (case b), 29 million (case c), 27 million (case d) and 32 million (case e) based on the original medium grid with 12 million mesh cells. Figure 20 shows a comparison of the time-averaged velocity streamline between combined drag reduction devices. Correspondingly, table 7 lists the center positions of the separation bubble. Obviously, the separation bubble has been weakened for most combinations. In Fig. 20(b), i.e. case (b), the separation bubble on the slant surface has significantly reduced while the center of the wake separation bubble has also moved away from the rear surface. Compared with Fig. 20(b), Fig. 20(c) shows a more significant improvement that the small separation bubble on the slant has disappeared. Besides, in the near-rear region, the position of the lower separation bubble center has significantly weakened and the formation of the upper vortex has been significantly affected, which will result in a greater increase in the rear surface pressure. For case (d), a significant change can also be observed in Fig. 20(d). The center of the separation bubble on the slant surface moves to the

top surface of the Ahmed body and the center of the wake vortex has moved away from the rear surface compared to the natural flow. Therefore, the drag reduction will increase compared to using riblets alone (Fig. 15b). For the last case, the small separation bubble on the slant is only slightly affected as shown in Fig. 20(e), and the formation of a large separation bubble in the near-rear region is suppressed a little. Thus, one can predict that the drag reduction effect is small.

Figure 21 shows a comparison of the surface pressure coefficient in the symmetrical plane for the four controlled flows and natural flow, which is similar to Fig. 12 and Fig. 19. The dashed line corresponds to the case (b) in which VGs are added to both the slant and rear surfaces. The dash-dot line, the long dashed line and the long dash-dot-dot line show cases (c), (d), and (e), respectively. For the dashed line, as discussed above, due to the flow disturbing effect of VGs on the slant surface, the $\mathrm{Cp}$ first decreases and then increases rapidly on the slant surface. At the rear surface, VGs also result in increased pressure coefficient, which is the same as 
X. Yang et al. / JAFM, Vol. 15, No. 1, pp. 193-207, 2022

Table 7 The position of wake vortex controlled by combined strategy, dimensions are given in millimeters

\begin{tabular}{|c|c|c|c|}
\hline \multirow{2}{*}{ Configurations } & \multicolumn{3}{|c|}{ X-direction position of the wake vortex core } \\
\cline { 2 - 4 } & Slant vortex & Rear vortex 1 & Rear vortex 2 \\
\hline Baseline Ahmed body & -144.53 & 58.01 & 82.22 \\
\hline $\begin{array}{c}\text { VGs added on both the slant } \\
\text { and rear surfaces }\end{array}$ & NONE & 86.63 & NONE \\
\hline $\begin{array}{c}\text { VGs added on the slant } \\
\text { surface with riblets added } \\
\text { on the rear surface }\end{array}$ & NONE & 64.59 & 80.78 \\
\hline $\begin{array}{c}\text { Riblets added on the slant } \\
\text { surface with VGs added on } \\
\text { the rear surface }\end{array}$ & NONE & 71.25 & 62.15 \\
\hline $\begin{array}{c}\text { Riblets added on both the } \\
\text { slant and rear surfaces }\end{array}$ & -151.78 & 48.14 & \\
\hline
\end{tabular}

Table 8 Comparison of drag coefficients with combined flow control

\begin{tabular}{|c|c|c|c|c|}
\hline Configurations & $\begin{array}{c}\text { Frictional drag } \\
\text { coefficients }\end{array}$ & $\begin{array}{c}\text { Frictional drag } \\
\text { reduction }\end{array}$ & $\begin{array}{c}\text { Total drag } \\
\text { coefficients }\end{array}$ & $\begin{array}{c}\text { Total drag } \\
\text { reduction }\end{array}$ \\
\hline Baseline Ahmed body & 0.0385 & - & 0.3401 & - \\
\hline $\begin{array}{c}\text { VGs added on both the slant } \\
\text { and rear surfaces }\end{array}$ & 0.0401 & $-4.16 \%$ & 0.3137 & $8.76 \%$ \\
\hline $\begin{array}{c}\text { VGs added on the slant } \\
\text { surface with riblets added } \\
\text { on the rear surface }\end{array}$ & 0.0352 & $8.57 \%$ & 0.3108 & $4.38 \%$ \\
\hline $\begin{array}{c}\text { Riblets added on the slant } \\
\text { surface with VGs added on } \\
\text { the rear surface }\end{array}$ & 0.0359 & $7.24 \%$ & 0.3252 & $3.41 \%$ \\
\hline $\begin{array}{c}\text { Riblets added on both the } \\
\text { slant and rear surfaces }\end{array}$ & 0.0329 & $14.55 \%$ & 0.3285 & $3.3 \%$ \\
\hline
\end{tabular}

using single VGs. In case (c), all separation bubbles are reduced. Therefore, the surface pressure is significantly increased, including both the slant and the rear surface. While for cases (d) and (e), riblets mainly contribute to the reduction of frictional resistance. Thus, only small pressure changes can be observed. When riblets are added to the slant surface and VGs are added to the rear surface, the effect of VGs seems to be interfered by the riblets, and the pressures obviously becomes small, which is even worse than the single VGs case (Fig. 12(b)).

Table 8 shows the total drag coefficients of the four cases using the combined strategy. Evidently, simultaneously adding drag reduction devices at the slant and rear surfaces can lead to better drag reduction effects than adding a single device on either the slant or rear surface. Adding different devices to different positions leads to different effects on the flow field and thus reduces the aerodynamic drag. The tribological performance is maximized for the combination of riblets on both the slant and rear surfaces, which is $14.55 \%$. In addition, it achieves a higher total drag reduction effect than a single riblet, which reaches $3.41 \%$. For the present study, adding a combination of VGs on the slant surface and riblets on the rear surface has the best total drag reduction effect and shows good tribological performance. The total drag reduction is $8.62 \%$ and the frictional drag reduction is $8.57 \%$.

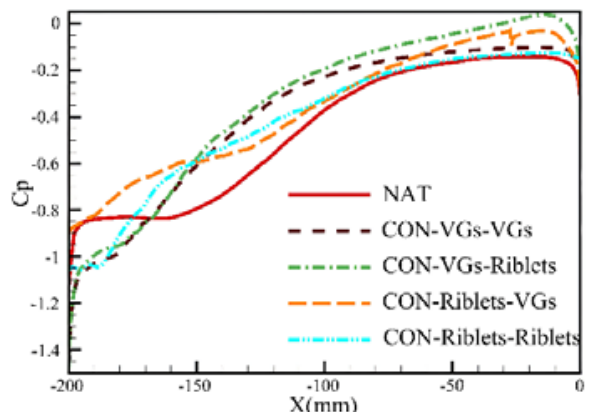

(a)

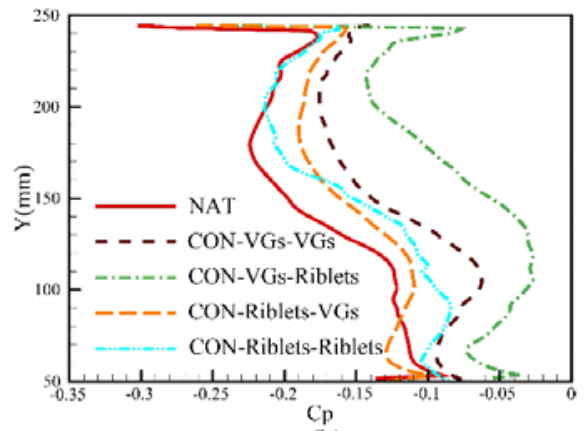

(b)

Fig. 21. Comparison of the surface pressure coefficient $C p$ between natural flow and the controlled flow with combined device: (a) Slant surface; (b) Rear surface. 
Contrarily, for the combination of riblets added on the slant surface and VGs added on rear surface, the effect of VGs on the rear surface is weakened due to the small vortices generated by the riblets. In return, the drag reduction effect is worse than using only VGs on the rear surface, yet still better than using only riblets.

\section{CONCLUSION}

The flow control for the Ahmed body at a $25^{\circ}$ slant is investigated here using the IDDES method. VGs and riblets are placed on the slant and rear surfaces of the Ahmed body to achieve passive flow control and reduce the aerodynamic drag. Based on the drag reduction effects of these two devices, different combined strategies were studied to maximize the flow control performance.

First, the unsteady external flow field of the Ahmed body at $25^{\circ}$ without flow control was studied using IDDES and verified with experiment data. The IDDES model successfully predicted a small vortices on the rear surface. The velocity profiles and pressures agree well with the experimental values. Then, two passive drag reduction devices were investigated, namely VGs and riblets. Small counter-rotating vortices were generated behind the near-wake region of the VGs and further developed downstream, which affected the formation of the large separation bubble. For the riblets, the nearwall spanwise flow was restricted by riblets. separation bubble on the slant surface and two large Low-speed flow is contained inside riblets and a second vortex is generated that impedes the momentum exchange and reduces the flow resistance. For each flow control strategy, either the formation of separation bubbles on the slant surface of the Ahmed body is suppressed or the separation near the rear region is delayed, which decreases the aerodynamic drag. The maximum drag reductions were $6.21 \%$ for VGs and $3.03 \%$ for riblets. Finally, a combined flow control method using both VGs and riblets was applied to the Ahmed body. By using different combinations of these two devices, significant increases in the frictional drag reduction and total drag reduction could be achieved compared to VGs or riblets alone. A maximum frictional drag reduction of $14.55 \%$ was observed for riblets on both the slant and rear. A total drag reduction of $8.62 \%$ was achieved when using VGs on the slant and riblets on the rear.

\section{ACKNOWLEDGEMENTS}

The work presented here was funded by NSFC funding 51775179, the authors is gratefully acknowledging this support.

\section{REFERENCES}

Ahmed, S. R., G. Ramm and G. Faltin (1984). Some salient features of the time-averaged ground vehicle wake. SAE Transactions, 473-503.

Aider, J. L., J. F. Beaudoin and J. E. Wesfreid (2010). Drag and lift reduction of a 3D bluff-body using active vortex generators. Experiments in Fluids 48(5), 771-789.

Altaf, A., A. A. Omar and W. Asrar (2014). Passive drag reduction of square back road vehicles. Journal of Wind Engineering and Industrial Aerodynamics 134, 30-43.

Anderson Jr, J. D. (2010). Fundamentals of aerodynamics. Tata McGraw-Hill Education.

Bao, Y. and J. Tao (2013). The passive control of wake flow behind a circular cylinder by parallel dual plates. Journal of Fluids and Structures 37, 201-219.

Beaudoin, J. F. and J. L. Aider (2008). Drag and lift reduction of a $3 \mathrm{D}$ bluff body using flaps. Experiments in Fluids 44(4), 491-501.

Capone, A. and G. P. Romano (2019). Investigation on the effect of horizontal and vertical deflectors on the near-wake of a square-back car model. Journal of Wind Engineering and Industrial Aerodynamics 185, 57-64.

Choi, H., W. P. Jeon and J. Kim (2008). Control of flow over a bluff body. Annual Review of Fluid Mechanics40, 113-139.

Choi, H., J. Lee, and H. Park (2014). Aerodynamics of heavy vehicles. Annual Review of Fluid Mechanics 46, 441-468.

Chok, C., S. Parameswaran, R. Sun and M. Gleason (1994). Numerical investigation of the effects of base slant on the wake pattern and drag of threedimensional bluff bodies with a rear blunt end. Journal of Wind Engineering and Industrial Aerodynamics 51(3), 269-285.

Conan, B., J. Anthoine and P. Planquart (2011). Experimental aerodynamic study of a car-type bluff body. Experiments in Fluids 50(5), 12731284.

Croll, R. H., W. T. Gutierrez, B. Hassan, J. E. Suazo and A. J. Riggins (1995). Experimental investigation of the ground transportation systems (GTS) project for heavy vehicle drag reduction (No. SAND-95-1337C; CONF960204-3). Sandia National Labs., Albuquerque, NM (United States).

Dean, B. and B. Bhushan (2012). The effect of riblets in rectangular duct flow. Applied Surface Science 258(8), 3936-3947.

Garcia-Mayoral, R. and J. Jiménez (2011). Drag reduction by riblets. Philosophical transactions of the Royal society A: Mathematical, Physical and Engineering Sciences 369(1940), 14121427.

Gilliéron, P. and A. Kourta (2010). Aerodynamic drag reduction by vertical splitter plates. Experiments in Fluids 48(1), 1-16.

Gritskevich, M. S., A. V. Garbaruk, J. Schütze and F. R. Menter (2012). Development of DDES and IDDES formulations for the k- $\omega$ shear stress transport model. Flow, Turbulence and 
Combustion 88(3), 431-449.

Grüneberger, R. and W. Hage (2011). Drag characteristics of longitudinal and transverse riblets at low dimensionless spacings. Experiments in Fluids 50(2), 363-373.

Guilmineau, E. (2008). Computational study of flow around a simplified car body. Journal of Wind Engineering and Industrial Aerodynamics 96(6-7), 1207-1217.

Guilmineau, E., G. Deng and J. Wackers (2011). Numerical simulation with a DES approach for automotive flows. Journal of Fluids and Structures 27(5-6), 807-816.

Guilmineau, E., G. B. Deng, A. Leroyer, P. Queutey, M. Visonneau and J. Wackers (2018). Assessment of hybrid RANS-LES formulations for flow simulation around the Ahmed body. Computers \& Fluids 176, 302-319.

Gutierrez, W. T., B. Hassan, R. H. Croll and W. H. W. H. Rutledge (1995). Aerodynamics overview of the ground transportation systems (GTS) project for heavy vehicle drag reduction (No. SAND-95-1339C; CONF-960204-2). Sandia National Labs., Albuquerque, NM (United States).

Han, T., V. Sumantran, C. Harris, T. Kuzmanov, M. Huebler, and T. Zak (1996). Flow-field simulations of three simplified vehicle shapes and comparisons with experimental measurements. SAE transactions, 820-835.

Han, X. and S. Krajnović (2013). Very large eddy simulation of passive drag control for a Dshaped cylinder. Journal of Fluids Engineering $135,8-16$.

Keogh, J., T. Barber, S. Diasinos and G. Doig (2016). The aerodynamic effects on a cornering Ahmed body. Journal of Wind Engineering and Industrial Aerodynamics 154, 34-46.

Krajnović, S. (2014). Large eddy simulation exploration of passive flow control around an Ahmed body. Journal of Fluids Engineering 136, 19-28.

Krajnović, S. and B. Basara (2010). LES of the flow around Ahmed body with active flow control. In Turbulence and Interactions (pp. 247-254). Springer, Berlin, Heidelberg.

Lam, K. and Y. F. Lin (2007). Drag force control of flow over wavy cylinders at low Reynolds number. Journal of Mechanical Science and Technology 21(9), 1331.

Lam, K., F. H. Wang and R. M. C. So (2004). Threedimensional nature of vortices in the near wake of a wavy cylinder. Journal of Fluids and Structures 19(6), 815-833.

Lienhart, H., C. Stoots and S. Becker (2003). Flow and turbulence structures in the wake of a simplified car model SAE transactions, 785796.
Minelli, G., S. Krajnović, B. Basara and B. R. Noack (2016). Numerical investigation of active flow control around a generic truck A-pillar. Flow, Turbulence and Combustion 97(4), 1235-1254.

Park, H., J. H. Cho, J. Lee, D. H. Lee and K. H. Kim (2013). Experimental study on synthetic jet array for aerodynamic drag reduction of a simplified car. Journal of Mechanical Science and Technology 27(12), 3721-3731.

Pujals, G., S. Depardon and C. Cossu (2010). Drag reduction of a 3D bluff body using coherent streamwise streaks. Experiments in Fluids 49(5), 1085-1094.

Rouméas, M., P. Gilliéron and A. Kourta (2009). Drag reduction by flow separation control on a car after body. International journal for Numerical Methods in Fluids 60(11), 12221240.

Schatzman, D., J. Wilson, E. Arad, A. Seifert and T. Shtendel (2014). Drag-reduction mechanisms of suction-and-oscillatory-blowing flow control. AIAA Journal 52(11), 2491-2505.

Selvaraju, P. N. and K. M. Parammasivam (2019). Empirical and Numerical Analysis of Aerodynamic Drag on a Typical SUV Car Model at Different Locations of Vortex Generator. Journal of Applied Fluid Mechanics 12(5), 1487-1496.

Serre, E., M. Minguez, R. Pasquetti, E. Guilmineau, G. B. Deng, M. Kornhaas, M. Schäfer, J. Fröhlich, C. Hinterberger, W. Rodi (2013). On simulating the turbulent flow around the Ahmed body: A French-German collaborative evaluation of LES and DES. Computers \& Fluids 78, 10-23.

Shankar, G. and G. Devaradjane (2018). Experimental and computational analysis on aerodynamic behavior of a car model with vortex generators at different yaw angles. Journal of Applied Fluid Mechanics 11(1), 285295.

Shur, M. L., P. R. Spalart, M. K. Strelets and A. K. Travin (2008). A hybrid RANS-LES approach with delayed-DES and wall-modelled LES capabilities. International Journal of Heat and Fluid Flow 29(6), 1638-1649.

Spalart, P. R. (1997). Comments on the feasibility of LES for wings, and on a hybrid RANS/LES approach. In Proceedings of first AFOSR international conference on DNS/LES. Greyden Press.

Spalart, P. R., S. Deck, M. L. Shur, K. D. Squires, M. K. Strelets and A. Travin (2006). A new version of detached-eddy simulation, resistant to ambiguous grid densities. Theoretical and Computational Fluid Dynamics 20(3), 181-195.

Spalart, P. R. and J. D. McLean (2011). Drag reduction: enticing turbulence, and then an industry. Philosophical Transactions of the Royal Society A: Mathematical, Physical and 
X. Yang et al. / JAFM, Vol. 15, No. 1, pp. 193-207, 2022

Engineering Sciences 369(1940), 1556-1569.

Walsh, M. and A. Lindemann (1984, January). Optimization and application of riblets for turbulent drag reduction. In 22nd aerospace sciences meeting (p. 347).

Walsh, M. J. (1983). Riblets as a viscous drag reduction technique. AIAA journal 21(4), 485486.

Wassen, E. and F. Thiele (2009). Road vehicle drag reduction by combined steady blowing and suction. In 39th AIAA Fluid Dynamics Conference (p. 4174).
Wood, R. M. (2006). A discussion of a heavy truck advanced aerodynamic trailer system. In Int. Symp. Heavy Veh. Weights Dimens., 9th, University Park, PA (pp. 1-14).

Zhang, Y., H. Chen, S. Fu and W. Dong (2018). Numerical study of an airfoil with riblets installed based on large eddy simulation. Aerospace Science and Technology 78, 661670.

Zou, L., C. Guo and C. Xiong (2013). Flow characteristics of the two tandem wavy cylinders and drag reduction phenomenon. Journal of Hydrodynamics 25(5), 737-746. 